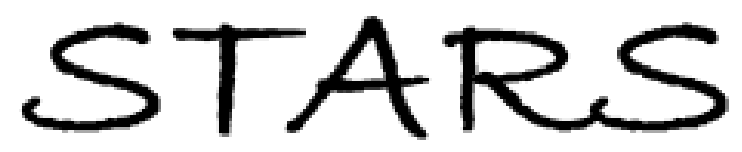

University of Central Florida

STARS

Faculty Bibliography 2000s

Faculty Bibliography

$1-1-2009$

\title{
Low temperature effects on the response time of liquid crystal displays
}

\author{
Linghui Rao \\ University of Central Florida \\ Sebastian Gauza \\ University of Central Florida \\ Shin-Tson Wu \\ University of Central Florida
}

Find similar works at: https://stars.library.ucf.edu/facultybib2000 University of Central Florida Libraries http://library.ucf.edu

This Article is brought to you for free and open access by the Faculty Bibliography at STARS. It has been accepted for inclusion in Faculty Bibliography 2000 s by an authorized administrator of STARS. For more information, please contactSTARS@ucf.edu.

\section{Recommended Citation}

Rao, Linghui; Gauza, Sebastian; and Wu, Shin-Tson, "Low temperature effects on the response time of liquid crystal displays" (2009). Faculty Bibliography 2000s. 2035.

https://stars.library.ucf.edu/facultybib2000/2035

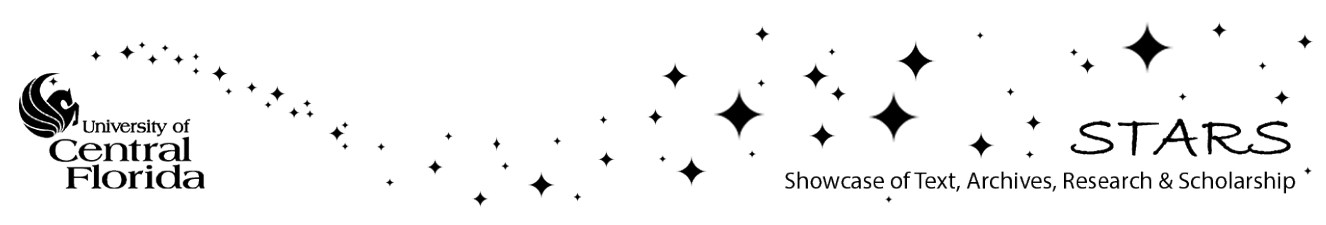




\section{Low temperature effects on the response time of liquid crystal displays}

Cite as: Appl. Phys. Lett. 94, 071112 (2009); https://doi.org/10.1063/1.3086883

Submitted: 15 January 2009 . Accepted: 02 February 2009 . Published Online: 19 February 2009

Linghui Rao, Sebastian Gauza, and Shin-Tson Wu

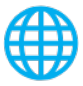

View Online

\section{ARTICLES YOU MAY BE INTERESTED IN}

Anchoring energy and cell gap effects on liquid crystal response time

Journal of Applied Physics 101, 103110 (2007); https://doi.org/10.1063/1.2734870

Temperature effect on liquid crystal refractive indices

Journal of Applied Physics 96, 19 (2004); https://doi.org/10.1063/1.1757034

Stabilization of the liquid crystal director in the patterned vertical alignment mode through formation of pretilt angle by reactive mesogen

Applied Physics Letters 90, 261910 (2007); https://doi.org/10.1063/1.2752105

\section{Applied Physics Letters}

Mid-IR and THz frequency combs special collection 


\title{
Low temperature effects on the response time of liquid crystal displays
}

\author{
Linghui Rao, Sebastian Gauza, and Shin-Tson Wu ${ }^{a}$ \\ College of Optics and Photonics, University of Central Florida, Orlando, Florida 32816, USA
}

(Received 15 January 2009; accepted 2 February 2009; published online 19 February 2009)

Low temperature effects on the liquid crystal response time for mobile displays are investigated. We studied the rotational viscosity and elastic constants of two commercial liquid crystal mixtures in the range from -20 to $60{ }^{\circ} \mathrm{C}$. At low temperatures, rotational viscosity still follows the modified Arrhenius law well but elastic constants are $\sim 30 \%-40 \%$ larger than those expected from mean-field theory. The responsible mechanism for this anomalous behavior originates from the increased fourth rank of order parameter at low temperatures. (C) 2009 American Institute of Physics. [DOI: $10.1063 / 1.3086883$ ]

Liquid crystal displays (LCDs) have been widely used in mobile devices, which require good performance under harsh ambient conditions, from indoor to outdoor and from hot to cold weather. Under strong anchoring energy condition, LC response time is mainly determined by the cell gap, viscoelastic coefficient $\left(\gamma_{1} / K_{i i}\right)$, where $\gamma_{1}$ is the rotational viscosity and $K_{i i}$ is the corresponding elastic constants of the LC alignment, and operating voltage. ${ }^{1-3}$ As the temperature decreases, $\mathrm{LC}$ response time increases dramatically because of the increased viscosity. Very few studies on the low temperature effects of LC response time have been reported. ${ }^{4}$

In this letter, low temperature behavior of LC response time is investigated. We measured the rotational viscosity and elastic constants of two commercial LC mixtures: (a) Merck MLC-6608 and (b) Slichem SLC-UCF-1 in the temperature range from -20 to $60{ }^{\circ} \mathrm{C}$. Some relevant physical properties of MLC-6608 (at $\sim 20^{\circ} \mathrm{C}$ ) are listed as follows: birefringence $\Delta n=0.083$ at $\lambda=589 \mathrm{~nm}$, dielectric anisotropy $\Delta \varepsilon=-4.2, \gamma_{1} \sim 186 \mathrm{mPa} \mathrm{s}, K_{33}=18.1 \mathrm{pN}$, and clearing temperature $T_{c} \sim 90{ }^{\circ} \mathrm{C}$, and for SLC-UCF-1 $\Delta n=0.124, \Delta \varepsilon$ $=3.2, \gamma_{1} \sim 60 \mathrm{mPa} \mathrm{s}$, and $T_{c} \sim 74.6{ }^{\circ} \mathrm{C}$. MLC-6608 is intended for vertical alignment, while SLC-UCF-1 is for twisted-nematic displays. ${ }^{5,6}$ Our results show that in this temperature range, the rotational viscosity follows the modified exponential law ${ }^{7,8}$ quite well, while the elastic constants deviate from mean-field theory ${ }^{9}$ by $\sim 30 \%-40 \%$. The increased elastic constant is favorable to LC response time.

Figures 1(a) and 1(b) show the measured rotational viscosity of MLC-6608 and SLC-UCF-1, respectively. The viscosity of MLC-6608 was measured with the electro-optic method described in Ref. 10, and SLC-UCF-1 was measured using a commercial instrument Instec ALCT-III. In Fig. 1, solid lines are fitting curves using the modified Arrhenius $\operatorname{model}^{7,8}$

$$
\gamma_{1}=b S \exp (E / K T),
$$

where $b$ is the proportionality constant, $S$ is the order parameter, $E$ is the activation energy of molecular rotation, and $K$ is the Boltzmann constant. The order parameter $(S)$ can be obtained by fitting the measured birefringence with Haller's semiempirical equations ${ }^{11,12}$

$$
\Delta n=(\Delta n)_{o} S
$$

and

$$
S=\left(1-T / T_{c}\right)^{\beta} .
$$

In Eqs. (2) and (3), $\Delta n_{o}$ is the birefringence at $T=0 \mathrm{~K}$ and $\beta$ is a material constant.

To fit the experimental data with Eq. (1), we use $\beta$ from temperature dependent birefringence measurement and leave $b$ and $E$ as adjustable parameters. The parameters we obtained are $b=9.51 \times 10^{-7} \mathrm{mPa} \mathrm{s}$ and $E=496.0 \mathrm{meV}$ for MLC-6608 $(\beta=0.20)$, and $b=1.96 \times 10^{-3} \mathrm{mPa} \mathrm{s}$ and
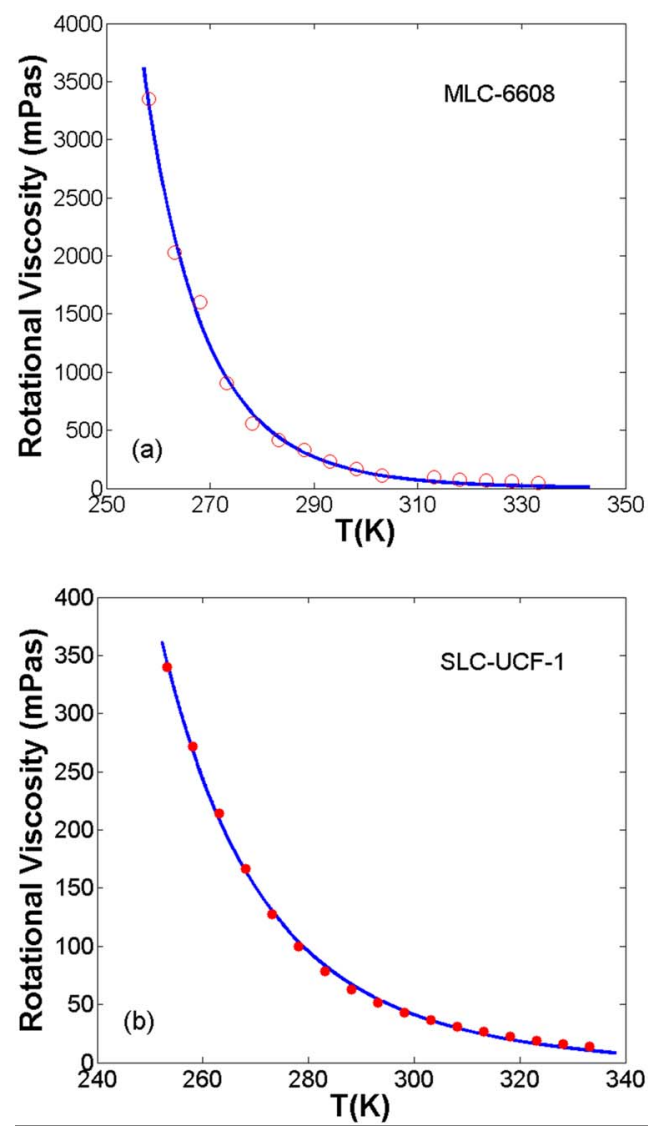

FIG. 1. (Color online) Measured rotational viscosity and fittings with Eq. (1) for (a) MLC-6608 and (b) SLC-UCF-1. 


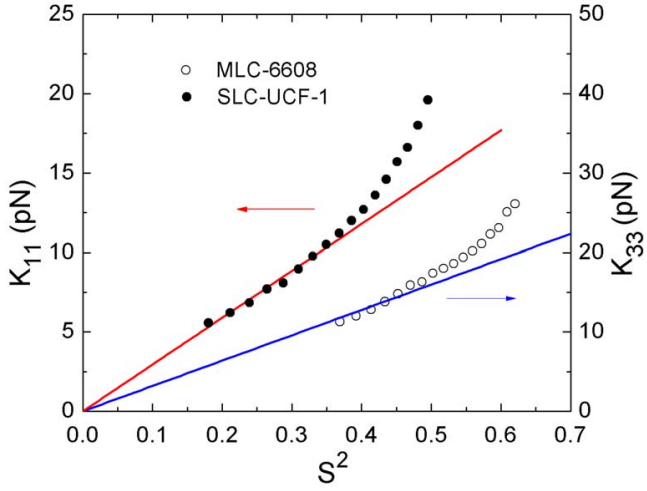

FIG. 2. (Color online) Measured elastic constants vs $S^{2}$ plot and linear fittings of MLC-6608 (open circles) and SLC-UCF-1 (filled circles).

$E=271.5 \mathrm{meV}$ for SLC-UCF-1 $(\beta=0.27)$. From Fig. 1, the fittings are pretty good and there are no unexpected behaviors at low temperatures for both mixtures.

Figure 2 shows the measured data and fittings with mean-field theory $\left(K_{i i} \sim S^{2}\right)$ of the elastic constants for the two LC mixtures studied from -20 to $60{ }^{\circ} \mathrm{C}$. The data were measured from a commercial instrument Instec ALCT-III based on threshold voltage and cell capacitance measurements. When doing the measurements, special attention should be paid to provide sufficient delay time between data acquisition, especially in the low temperature region where the $\mathrm{LC}$ response time is fairly slow $(\sim 1 \mathrm{~s})$.

From Maier-Saupe theory (commonly referred as meanfield theory $)^{9}$ the elastic constant is related to the nematic order parameter $(S)$ as

$$
K_{i i}=A_{o} S^{2},
$$

where $A_{o}$ is a material constant. As shown in Fig. 2, our results agree with mean-field theory well in the high temperature side. As the temperature decreases, the deviation is gradually amplified for both mixtures studied. At $-20{ }^{\circ} \mathrm{C}$, the $K_{33}$ for MLC-6608 and $K_{11}$ for SLC-UCF-1 are about $30 \%-40 \%$ larger than those expected from mean-field theory.

To explain this anomalous behavior, we consider a model proposed by Gelbart and Ben-Shaul (referred as GB model), ${ }^{13}$

$$
K_{i i}=k_{i i}^{22} \bar{P}_{2} \bar{P}_{2}+k_{i i}^{24} \bar{P}_{2} \bar{P}_{4},
$$

where $k_{i i}^{22}$ and $k_{i i}^{24}$ are linearly dependent on the temperature, and $\bar{P}_{2}$ and $\bar{P}_{4}$ are the second and fourth rank of the order parameter. With $k_{i i}^{22}=A_{i i} T$ and $k_{i i}^{24}=B_{i i} T$, we can rewrite Eq. (5) as

$$
\mathrm{K}_{i i}=\mathrm{A}_{i i} T S^{2}+B_{i i} T S \bar{P}_{4} .
$$

The temperature dependence of $K_{i i} /\left(\bar{P}_{2}\right)^{2}$ is determined primarily by the relative magnitude and sign of $\bar{P}_{4}$. As the temperature increases, more fluctuations on the LC directors are induced by the higher thermal energy. As a result, the LC directors are more randomly distributed, which causes the order parameters, both $\bar{P}_{2}$ and $\bar{P}_{4}$, to gradually decrease. In general, $\bar{P}_{4}$ is a complicated function of temperature and no analytical form is found. According to Ref. $14, \bar{P}_{4}$ can be approximated as
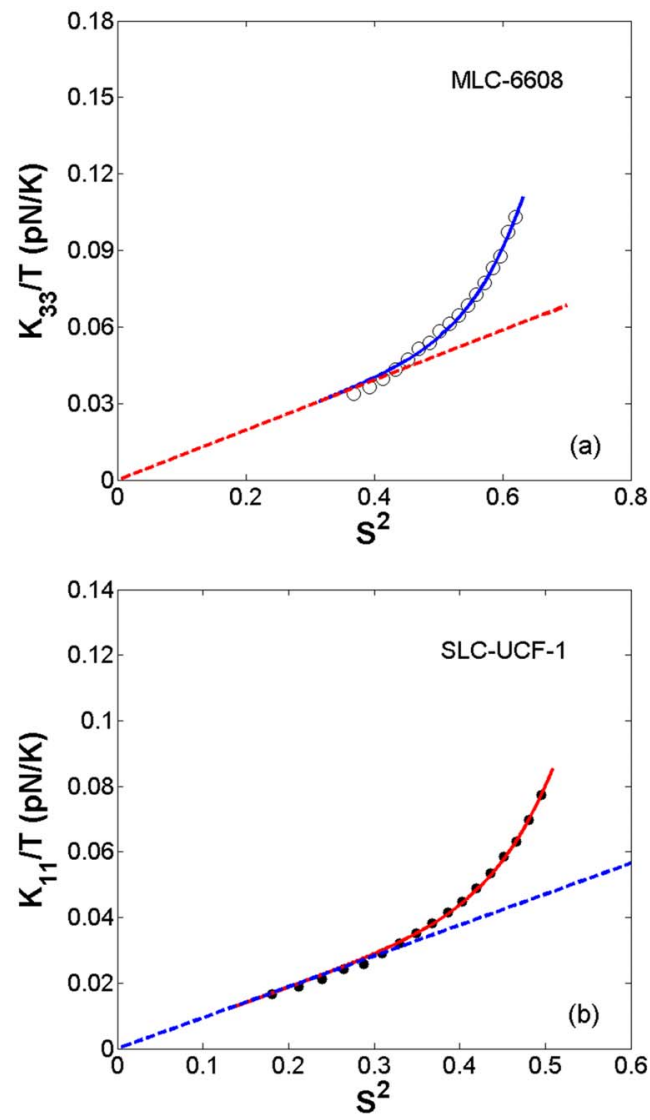

FIG. 3. (Color online) Measured $K_{i i} / T$ vs $S^{2}$ and fittings of (a) MLC-6608 and (b) SLC-UCF-1, solid lines are fittings from Eqs. (6) and (7), dashed lines are the linear fittings.

$$
\bar{P}_{4}=\left(1-T / T_{c}\right)^{\alpha} .
$$

To fit the experimental data with Eqs. (6) and (7), we use $S$ extracted from birefringence data through fitting Eq. (2), while leaving $A_{i i}, B_{i i}$, and $\alpha$ as adjustable parameters. If we treat $B_{i i} \bar{P}_{4}$ as a whole, then there will be only two adjustable parameters. Here, we use three in order to better explain the temperature dependence of $\bar{P}_{4}$.

Through fitting, we found $A_{33}=0.0977 \mathrm{pN} / \mathrm{K}, B_{33}$ $=2.1680 \mathrm{pN} / \mathrm{K}$, and $\alpha=3.0914$ for MLC-6608, and $A_{11}$ $=0.0942 \mathrm{pN} / \mathrm{K}, \quad B_{11}=5.6584 \mathrm{pN} / \mathrm{K}$, and $\alpha=3.7341$ for SLC-UCF-1. The fittings (solid lines) match well with the experimental data in Fig. 3, especially in the low temperature region where the data have deviated from mean-filed theory. Among the three adjustable parameters, $A_{i i}$ is more sensitive than $B_{i i}$ and $\alpha$ because the second term in Eq. (6) is generally smaller than the first term. As shown in Fig. 3, the dashed straight lines for both mixtures show that aside from the low temperature region, it is still a linear relation because the $B \bar{P}_{4}$ term is negligible. Therefore, in this temperature region, Eq. (6) can be simplified as

$$
K_{i i} \approx A_{i i} T S^{2} \text {, }
$$

with the fitted parameters $A_{33}=0.0977 \mathrm{pN} / \mathrm{K}$ for MLC-6608 and $A_{11}=0.0942 \mathrm{pN} / \mathrm{K}$ for SLC-UCF-1, respectively.

In the nematic range, the oversimplified Eq. (7) implies that $\bar{P}_{4}$ is always positive. However, it has been reported that $\bar{P}_{4}$ value, determined by means of resonance Raman measurements on $\beta$-carotene dissolved as an orientational probe 


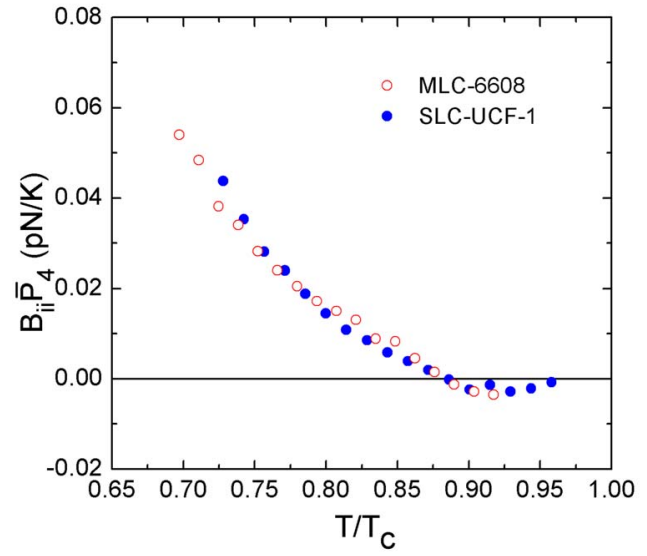

FIG. 4. (Color online) $B_{i i} \bar{P}_{4}$ vs $T / T_{c}$ for MLC-6608 (open circles) and SLC-UCF-1 (filled circles).

for the single compound 5CB, can become slightly negative on the high temperature side. ${ }^{14,15}$ Without using the approximation in Eq. (7), if we rewrite Eq. (6) as

$$
B_{i i} \bar{P}_{4}=\frac{K_{i i}-A_{i i} T S^{2}}{T S}
$$

we can obtain the $B_{i i} \bar{P}_{4}$ value once the $A_{i i}$ has been determined from Eq. (8). Unlike the single compound mentioned above, the $\bar{P}_{4}$ of our two commercial LC mixtures cannot be directly determined, neither are there any effective approaches to get $B_{i i}$ in the nematic range. Nevertheless, the plot of $B_{i i} \bar{P}_{4}$ versus $T / T_{c}$ in Fig. 4 represents the same trend as $\bar{P}_{4}$ versus $T / T_{c}$ because $B_{i i}$ is a constant. In the high temperature range, the $\bar{P}_{4}$ term is small and slightly negative. This is in good agreement with the experimental data reported in Ref. 15 for $5 \mathrm{CB}$, and explains well that in high temperature region the fittings from Eqs. (6) and (7) are slightly larger than our experimental data. Also from Fig. 4, we find that $\bar{P}_{4}$ in low temperature region increases rapidly for both LC mixtures studied.

The GB model, which includes the $\bar{P}_{4}$ and $T$ terms, can explain well why the measured $K_{i i}$ value is $\sim 1.3-1.4 \mathrm{x}$ higher at $-20{ }^{\circ} \mathrm{C}$ than that predicted by Maier-Saupe theory for MLC-6608 and SLC-UCF-1. In a limited temperature range, the change of $T$ is much smaller than that of the $S^{2}$ term so that $k_{i i}^{22}$ and $k_{i i}^{24}$ can be treated as constants. Under this condition, Eq. (8) is reduced to Maier-Saupe theory.

In conclusion, response time in low temperature region is important for mobile LCDs. Our experimental results show that, as the temperature decreases, the rotational viscosity still follows the exponential law quite well, but the elastic constants increase faster than expected from meanfield theory. To explain this anomalous behavior, the contribution of $\bar{P}_{4}$ needs to be taken into consideration. The enhanced elastic constant is favorable for improving the response time of mobile displays at low temperatures.

The authors are indebted to Professor X. Liang for providing us the TN mixture and Chi-Mei Optoelectronics (Taiwan) for financial support.

${ }^{1}$ E. Jakeman and E. P. Raynes, Phys. Lett. A 39, 69 (1972).

${ }^{2}$ S. T. Wu, Appl. Opt. 28, 48 (1989).

${ }^{3}$ M. Jiao, Z. Ge, Q. Song, and S. T. Wu, Appl. Phys. Lett. 92, 061102 (2008)

${ }^{4}$ J. Li, S. Gauza, and S. T. Wu, J. Appl. Phys. 96, 19 (2004).

${ }^{5}$ M. Schadt and W. Helfrich, Appl. Phys. Lett. 18, 127 (1971).

${ }^{6}$ S. T. Wu and C. S. Wu, Appl. Phys. Lett. 68, 1455 (1996).

${ }^{7}$ W. H. De Jeu, Mol. Cryst. Liq. Cryst. 63, 83 (1981).

${ }^{8}$ S. T. Wu and C. S. Wu, Liq. Cryst. 8, 171 (1990).

${ }^{9}$ W. Maier and A. Saupe, Z. Naturforsch. Teil A 14, 882 (1959); 15, 287 (1960).

${ }^{10}$ S. T. Wu and C. S. Wu, Phys. Rev. A 42, 2219 (1990).

${ }^{11}$ I. Haller, Prog. Solid State Chem. 10, 103 (1975).

${ }^{12}$ S. T. Wu, Phys. Rev. A 33, 1270 (1986).

${ }^{13}$ W. M. Gelbart and A. Ben-Shaul, J. Chem. Phys. 77, 916 (1982).

${ }^{14}$ S. Kobinata, Y. Nakajima, H. Yoshida, and S. Maeda, Mol. Cryst. Liq. Cryst. 66, 67 (1981).

${ }^{15}$ K. Miyano, J. Chem. Phys. 69, 4807 (1978). 